\title{
ANÁLISE DA ESTRUTURA ARBUSTIVO-ARBÓREA DE UMA ÁREA DE CERRADO Sensu Stricto, MUNICÍPIO DE AQUIDAUANA-MATO GROSSO DO SUL ${ }^{1}$
}

\author{
Bruna Gardenal Fina² e Reinaldo Monteiro ${ }^{3}$
}

\begin{abstract}
RESUMO - Este trabalho teve como objetivo registrar a composição florística e estrutura de uma área de Cerrado stricto senso, com ênfase no estrato arbustivo-arbóreo. Foram instaladas 30 parcelas de 10 x 10 m, distribuídas em blocos de 10 parcelas contíguas, onde os indivíduos com PAP superior ou igual a $10 \mathrm{~cm}$ foram incluídos. O número total de indivíduos amostrados foi de 1.107, pertencentes a 33 famílias e 88 espécies, evidenciando-se alta riqueza. A área basal total e a densidade total foram, respectivamente, de 6,651 m² e 3.690 indivíduos por hectare. Os valores de diversidade (3.868 nats/indivíduo) e equabilidade $(0,86)$ indicaram grande heterogeneidade e baixa dominância ecológica. As espécies mais importantes foram Qualea grandiflora Mart., Lafoensia pacari St. Hil., Magonia pubescens St. Hil e Qualea parviflora Mart., que juntas representaram 57,7\% do VI
\end{abstract}

Palavras-chave: Cerrado; Fitossociologia; Mato Grosso do Sul.

\section{A WOODY COMMUNITY STRUCTURE IN A CERRADO Sensu Stricto AREA, MUNICIPALITY OF AQUIDAUANA-MATO GROSSO DO SUL}

\begin{abstract}
The objective of this work was to study the floristic composition and structure of the Cerrado (Brazilian savannah), focusing on the tree and shrub strata. A total of 30 plots (10x10m) were set up, distributed in 10 contiguous blocks where individuals with a minimum PBH of $10 \mathrm{~cm}$ were sampled. A total of 1,107 individuals were sampled. Such individuals belonged to 33 families and 88 species, showing high values of richness. Total basal area and total density were respectively $6,651 \mathrm{~m}^{2}$ and 3690 individuals per hectare. The values of diversity (3.868 nats/individual) and evenness (0.86) show high heterogeneity and low ecological dominance. The most important species were Qualea grandiflora Mart., Lafoensia pacari St. Hil., Magonia pubescens St. Hil. and Qualea parviflora Mart., which together represented 57.7\% of VI.
\end{abstract}

Keywords: Cerrado; fitossocioly; Mato Grosso do Sul State, Brazil.

\section{INTRODUÇÃO}

O Cerrado localiza-se predominantemente no Planalto Central do Brasil e constitui a segunda maior formação vegetal brasileira, ocupando aproximadamente dois milhões de $\mathrm{km}^{2}$ da região do Brasil Central e cerca de 23\% do território nacional (RATTER et al., 1997).

A expansão das fronteiras agrícolas, a partir da década de 1970, fez com que grande parte da cobertura vegetal nativa em todo o país fosse substituída por áreas de cultivos e pastagens. No Centro-Oeste, hoje aproximadamente $67 \%$ das áreas de Cerrado estão altamente modificadas, com presença de voçorocas e assoreamentos (IBAMA, 2002).

Essa situação é bastante preocupante, uma vez que existem estimativas da perda de 9,75 milhões de hectares de áreas de Cerrado por ano no Brasil (AGUIAR et al., 2004). Até o ano 2002, a estimativa era de que aproximadamente 55\% de sua área original já havia sido destruída, e nesse período a região Leste do Estado de Mato Grosso do Sul destacou-se, juntamente com porções dos Estados de Goiás, Tocantins, Bahia e Minas Gerais, por apresentarem grandes áreas de desmatamentos (MACHADO et al., 2004).

\footnotetext{
${ }^{1}$ Recebido em 07.07.2008 aceito para publicação em 25.06.2013.

${ }^{2}$ Universidade Federal de Mato Grosso do Sul, UFMS, Brasil. E-mail: <bruna.fina@ufms.br>.

${ }^{3}$ Universidade Estadual Paulista, UNESP, Brasil. Email: <reimonte@rc.unesp.br>.
} 
Silva et al. (2000), quantificando as classes de vegetação do Pantanal através de levantamento aéreo, constataram que, na sub-região de Aquidauana, apenas 20,2\% correspondiam à fisionomia de Cerrado stricto senso, enquanto $31,2 \%$ da vegetação correspondia à fisionomia de Cerradão e apenas 2,9\%, às de Matas Semidecíduas.

O Município de Aquidauana localiza-se em uma área de transição entre o planalto e a planície pantaneira, possuindo, ainda, vários fragmentos de vegetação nativa, constituídos, principalmente, por vegetação de Savana em suas diferentes fisionomias. Os inventários florísticos locais são escassos. Há levantamentos macrorregionais realizados pelos Projetos Radam Brasil (BRASIL, 1990), PCBAP (BRASIL, 1997) e Embrapa (1998), que norteiam a classificação da vegetação em geral.

Os estudos fitossociológicos na região do Pantanal iniciaram-se a partir da década de 1980, englobando diferentes fitofisionomias (Cerrado stricto senso, Cerradão e florestas), em ambos os Estados (Mato Grosso e Mato Grosso do Sul), porém com poucos trabalhos sobre o Cerrado stricto senso no Estado de Mato Grosso do Sul.

Dessa forma, este estudo teve como objetivo analisar a composição e estrutura da vegetação na fisionomia de Cerrado stricto senso, no intuito de verificar se, por representar uma área de transição planaltoplanície pantaneira, existe maior variação em sua composição florística e estrutural, além de fornecer informações que poderão subsidiar futuros programas de manejo e conservação da área.

\section{MATERIAL E MÉTODOS}

\subsection{Caracterização da área de estudo}

O município de Aquidauana localiza-se à noroeste do Estado, entre os paralelos 18 $48^{\prime} 15^{\prime}$ ' e 20²8'57' 'S e 54 55' 45' ' e 56 59'15'’ W, na depressão do Rio Paraguai.

O local de estudo pertence à Universidade Estadual de Mato Grosso do Sul (UEMS) e situa-se a $12 \mathrm{~km}$ da

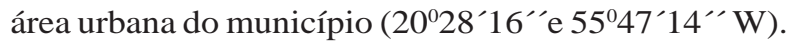
A Fazenda apresenta relevo esculpido em rochas areníticas e possui 806 ha, com aproximadamente 300 ha ocupados por áreas de pastagens e 160 ha da área de preservação permanente, além das áreas com construções prediais e cultivos. Localiza-se na região da Serra de Maracajú e nela ocorre contato savanafloresta estacional, representando uma área de grande beleza cênica.

O clima foi classificado como Awa, segundo Koeppen, ou seja, tropical megatérmico, em que a temperatura média do mês mais frio é superior a $18^{\circ} \mathrm{C}$, com inverno seco e verão chuvoso. A temperatura média anual foi de $24,6^{\circ} \mathrm{C}$ e a precipitação média anual foi de $1.331,04$ $\mathrm{mm}$. O solo foi classificado como Latossolo Vermelho, textura arenosa, com solo ácido, álico e distrófico. A altimetria varia entre 300 e $600 \mathrm{~m}$, e os principais litotipos relacionam-se à bacia Sedimentar do Paraná, representados localmente pelas Formações Furnas, Aquidauana e Ponta Grossa (BRASIL, 1997).

Historicamente, a área já sofreu ações antrópicas no passado, principalmente com a presença de gado e retirada constante de madeira.

\subsection{Levantamento fitossociológico}

O método utilizado no estudo fitossociológico foi o de parcelas (MUELLER-DOMBOIS; ELLENBERG, 1974). O critério de inclusão foi o de indivíduos lenhosos com no mínimo $10 \mathrm{~cm}$ de PAP (perímetro à altura do peito), medido a 1,30 m do solo. Os indivíduos mortos em pé foram considerados no estudo da estrutura da comunidade. As coletas e marcações foram realizadas entre março de 2005 e agosto de 2006, sendo mensal o acompanhamento na área.

Os indivíduos amostrados receberam placa metálica com numeração sequencial e foram feitas as anotações de campo (data, local, altura, diâmetro, hábito, cor, odor, presença de espinhos nos ramos e látex, entre outras). Posteriormente, esses foram coletados, prensados, secos em estufa e identificados. O sistema de classificação adotado foi o de Cronquist (1981), exceto para a família Leguminosae. O material fértil foi incluído no Herbarium Rioclarense (HRCB).

Foram instaladas 30 parcelas de $10 \times 10$ m cada, distribuídas em três blocos de 10 parcelas contíguas (50 x 20), distantes no mínimo 20 m da margem da vegetação. A localização dos blocos de parcelas foi definida por meio de observações em campo.

Para o cálculo dos parâmetros fitossociológicos, utilizou-se o Programa FITOPAC-1 (SHEPHERD, 1994), em que são considerados os parâmetros relativos de Densidade, Frequência, Dominância e Valor de Importância das espécies. 
Para verificar a diversidade da área estudada, utilizou-se o Índice de Shannon ( $\mathrm{H}^{\prime}$ ) baseado na abundância proporcional das espécies. A equabilidade foi obtida pelo índice de Pielou ( $\left.\mathrm{J}^{\prime}\right)$. A análise da similaridade qualitativa entre as parcelas foi realizada utilizando-se o coeficiente de Jaccard (J).

\section{RESULTADOS E DISCUSSÃO}

A suficiência amostral foi analisada através da curva espécie-área (Figura 1). Esta se estabilizou na $20^{\mathrm{a}}$ parcela, não ocorrendo acréscimo de novas espécies nas últimas 10 parcelas analisadas. Assim, o tamanho da área amostrada foi suficiente para a comparação da composição florística e da estrutura dessa fisionomia.

Foram amostrados o total 1.107 indivíduos, pertencentes a 33 famílias e 88 espécies (Tabela 1). O número de espécies encontradas está de acordo com os padrões de riqueza para o estrato lenhoso das áreas de Cerrado stricto senso, não ultrapassando 120 espécies (RATTER et al., 1997). Entretanto, na Região CentroOeste esse número pode ser considerado alto, uma vez que Ratter et al. (2003) registraram uma média de 58 espécies no Estado de Mato Grosso do Sul, 79 espécies em Goiás, 65 em Mato Grosso e 72 em Tocantins.

A diversidade $\left(\mathrm{H}^{\prime}\right)$ foi de 3,86 e a equabilidade, 0,86. É possível observar, na Tabela 2, que o valor de diversidade está acima da média dos valores encontrados nas áreas de Cerrado stricto senso (FELFILI et al., 1993; SILVEIRA et al., 2000; FELFILI et al., 2002; SILVA et al., 2002; BALDUÍNO et al., 2005; BORGES; SHEPHERD, 2005; MARIMON JR.; HARIDASAN, 2005;

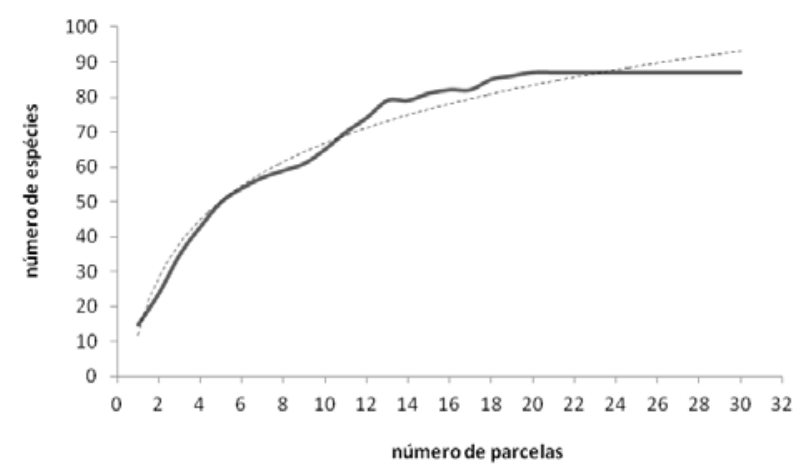

Figura 1 - Curva espécie-área de uma área de cerrado stricto senso no município de Aquidauana-MS.

Figure 1 - Species-area curve of Cerrado senso stricto in Aquidauana-MS.
MEDEIROS et al., 2005; NERI et al., 2007). Esse fato pode estar associado à presença de diferentes fitofisionomias (Mata Semidecídua e Cerradão) em locais bastante próximos, permitindo que espécies não muito frequentes nas áreas de Cerrado ali ocorram.

A equabilidade $\left(\mathrm{J}^{`}\right)$ foi semelhante às outras áreas analisadas (Tabela 2) e pode ser considerada alta, indicando que as espécies estão bem distribuídas nas áreas de Cerrado stricto senso de modo geral. Entretanto, a análise associada do índice de Shannon e da equabilidade demonstra a maior diversidade da área de estudo em relação aos demais trabalhos analisados devido à maior uniformidade na distribuição do número de indivíduos das espécies.

A densidade total foi de 3.690 indivíduos por hectare, e a área basal total foi de $6,65 \mathrm{~m}^{2}$, valores esses também acima da média daqueles encontrados em outras áreas de Cerrado stricto senso, exceto em Senador Modestino Gonçalves (Tabela 2), que se encontra em regeneração. A alta densidade da área amostrada é corroborada pela observação de que esta se encontra em processo de recuperação de ações antrópicas passadas, principalmente relacionadas à retirada de madeira, ação do fogo e pisoteamento pelo gado, uma vez que atualmente essas atividades estão sendo coibidas no local, o que possibilita a sua recuperação natural.

Os indivíduos mortos em pé representaram 5,6\% do total amostrado e 6,3\% da área basal, ocupando primeira posição em valor de importância (VI). Esses valores estão próximos aos encontrados em outros estudos nas áreas de Cerrado stricto senso, onde a quantidade de indivíduos mortos variou entre 5 e 14\%, dependendo, principalmente, da ocorrência de fogo (FELFILI et al., 2001; BALDUÍNO et al., 2005; MARIMON JR.; HARIDASAN, 2005).

As famílias Vochysiaceae, Fabaceae, Myrtaceae, Erythroxylaceae, Caesalpinaceae e Lythraceae apresentaram os maiores valores de valores de importância, perfazendo 52,34\% do total. A família mais importante foi Vochysiaceae (VI = 14,56\%), com alta frequência ( $F R=26,3 \%$ ) e densidade ( $D R=14,81$ ), sendo comum seu predomínio em áreas de Cerrado (FELFILI et al., 1993; SILVA et al., 2002; BALDUÍNO et al., 2005; NERI et al., 2007), uma vez que suas espécies são capazes de acumular alumínio (GOODLAND, 1970) e, geralmente, apresentam elevada biomassa (FELFILI et al., 2002; SILVA et al., 2002).

Revista Árvore, Viçosa-MG, v.37, n.4, p.577-585, 2013 
Tabela 1 - Parâmetros fitossociológicos das espécies amostradas na área de cerrado stricto senso, município de Aquidauana-MS, em ordem decrescentes de VI, com respectivos $n^{\circ}$ do coletor (Fina, B.G.).

Table 1 - Phytosociological parameters of sampled species of Cerrado senso stricto in Aquidauana-MS, in decreasing order of VI, with their respective collector numbers.

\begin{tabular}{|c|c|c|c|c|c|c|c|c|}
\hline Espécies & $\mathrm{Ni}$ & $\mathrm{Na}$ & DR & DoR & FR & $\begin{array}{l}\text { Altura } \\
\text { média }\end{array}$ & $\begin{array}{l}\text { Diâmetro } \\
\text { médio }\end{array}$ & VI \\
\hline Mortas & 62 & 26 & 5.60 & 6.36 & 4.56 & 0,0 & 8,1 & 16.52 \\
\hline Qualea grandiflora Mart. (390) & 49 & 24 & 4.43 & 7.03 & 4.21 & 4,4 & 9,4 & 15.67 \\
\hline Lafoensia pacari A. St. -Hil. (302 ) & 69 & 20 & 6.23 & 5.75 & 3.51 & 3,8 & 7,9 & 5.50 \\
\hline Magonia pubescens A. St.- Hil. (426) & 72 & 12 & 6.50 & 5.24 & 2.11 & 4,3 & 7,0 & 13.85 \\
\hline Qualea parviflora Mart. (228) & 39 & 19 & 3.52 & 5.82 & 3.33 & 4,9 & 10,2 & 12.68 \\
\hline Erythroxylum deciduum A. St.- Hil. (396) & 59 & 18 & 5.33 & 3.63 & 3.16 & 2,3 & 5,7 & 12.12 \\
\hline Callisthene fasciculata Mart. (305) & 40 & 18 & 3.61 & 4.82 & 3.16 & 4,9 & 9,3 & 11.59 \\
\hline Myrcia fallax (Rich.) DC. (256) & 46 & 19 & 4.16 & 3.72 & 3.33 & 3,9 & 7,3 & 11.21 \\
\hline Erythroxylum suberosum A. St.-Hil. (382) & 49 & 21 & 4.43 & 2.13 & 3.68 & 2,5 & 5,6 & 10.24 \\
\hline Callisthene minor Mart. (300) & 36 & 18 & 3.25 & 3.50 & 3.16 & 5,1 & 8,2 & 9.91 \\
\hline Acosmium subelegans (Mohlenbr.) Yakovlev & 37 & 16 & 3.34 & 2.65 & 2.81 & 4,0 & 7,3 & 8.80 \\
\hline Hymenaea stigonocarpa Mart. ex Hayne (220) & 23 & 12 & 2.08 & 3.90 & 2.11 & 4,4 & 8,9 & 8.08 \\
\hline Myrcia sp2 & 29 & 11 & 2.62 & 2.14 & 1.93 & 3,8 & 7,2 & 6.69 \\
\hline Dalbergia miscolobium Benth. & 31 & 10 & 2.80 & 1.81 & 1.75 & 3,8 & 6,5 & 6.37 \\
\hline Diptychandra aurantica Tul. (299) & 11 & 9 & 0.99 & 3.06 & 1.58 & 4,9 & 11,8 & 5.64 \\
\hline Byrsonima coccolobifolia Kunth. (246) & 17 & 12 & 1.54 & 1.03 & 2.11 & 3,3 & 6,4 & 4.67 \\
\hline Agonandra brasiliensis Miers ex Benth. \& Hook. f. & 16 & 11 & 1.45 & 1.26 & 1.93 & 4,4 & 7,6 & 4.63 \\
\hline Alibertia edulis (Rich.) A. Rich. ex DC. (303) & 17 & 11 & 1.54 & 1.16 & 1.93 & 3,4 & 6,6 & 4.63 \\
\hline Kielmeyera rubriflora Cambess (242) & 16 & 11 & 1.45 & 1.14 & 1.93 & 3,8 & 7,0 & 4.51 \\
\hline Sthrychnos pseudoquina A. St.- Hil. (308) & 18 & 10 & 1.63 & 0.88 & 1.75 & 3,4 & 7,1 & 4.26 \\
\hline Tabebuia ochracea (Cham.) Standl. & 20 & 9 & 1.81 & 0.67 & 1.58 & 2,7 & 5,0 & 4.06 \\
\hline \multicolumn{9}{|l|}{ Pseudobombax longiflorum (Mart. \& Zuch.) } \\
\hline A. Robyns (316) & 10 & 9 & 0.90 & 1.37 & 1.58 & 2,9 & 8,2 & 3.85 \\
\hline Davilla elliptica A. St. -Hil. (298) & 19 & 7 & 1.72 & 0.86 & 1.23 & 2,0 & 5,8 & 3.81 \\
\hline Pterodon emarginatus Vogel (413) & 12 & 6 & 1.08 & 1.65 & 1.05 & 5,5 & 10,3 & 3.79 \\
\hline Albizia niopoides (Spruce ex Benth.) Burkart & 7 & 6 & 0.63 & 2.00 & 1.05 & 7,1 & 14,7 & 3.68 \\
\hline Xylopia aromatica (Lam.) Mart. (237) & 15 & 8 & 1.36 & 0.85 & 1.40 & 4,9 & 6,2 & 3.61 \\
\hline \multicolumn{9}{|l|}{ Tabebuia aurea (Silva Manso) Benth. \& } \\
\hline Hook. f. ex S. Moore & 10 & 8 & 0.90 & 1.17 & 1.40 & 3,9 & 8,4 & 3.4 \\
\hline Andira cujabensis Benth. (325) & 12 & 8 & 1.08 & 0.86 & 1.40 & 4,2 & 7,1 & 3.3 \\
\hline Dimorphandra mollis Benth. & 10 & 9 & 0.90 & 0.84 & 1.58 & 4,5 & 7,9 & 3.32 \\
\hline Plathymenia reticulata Benth. & 8 & 6 & 0.72 & 1.44 & 1.05 & 5,0 & 11,2 & 3.22 \\
\hline Combretum leprosum Mart. (368) & 7 & 7 & 0.63 & 1.25 & 1.23 & 6,3 & 11,9 & 3.11 \\
\hline Aspidosperma tomentosum Mart. (216) & 10 & 7 & 0.90 & 0.67 & 1.23 & 3,5 & 6,6 & 2.80 \\
\hline Anadenanthera colubrina (Vell.) Brenan (219) & 7 & 5 & 0.63 & 1.24 & 0.88 & 5,1 & 11,3 & 2.75 \\
\hline Himatanthus obovatus (Müll. Arg.) Woodson (217) & 7 & 7 & 0.63 & 0.88 & 1.23 & 4,6 & 9,0 & 2.74 \\
\hline Genipa americana L. & 11 & 7 & 0.99 & 0.45 & 1.23 & 3,0 & 5,4 & 2.68 \\
\hline Annona crassifolia Mart. (309) & 10 & 7 & 0.90 & 0.47 & 1.23 & 3,4 & 5,9 & 2.60 \\
\hline Centrolobium sp. & 10 & 7 & 0.90 & 0.45 & 1.23 & 4,0 & 5,8 & 2.58 \\
\hline Curatella americana L. (332) & 6 & 4 & 0.54 & 1.26 & 0.70 & 5,4 & 12,2 & 2.50 \\
\hline Casearia sylvestris Sw. (323) & 13 & 6 & 1.17 & 0.25 & 1.05 & 2,8 & 4,0 & 2.48 \\
\hline Sclerolobium paniculatum Vogel & 7 & 6 & 0.63 & 0.70 & 1.05 & 3,7 & 7,7 & 2.39 \\
\hline Dipteryx alata Vogel & 8 & 4 & 0.72 & 0.88 & 0.70 & 4,9 & 8,5 & 2.30 \\
\hline Buchenavia tomentosa Eichler (297) & 6 & 5 & 0.54 & 0.87 & 0.88 & 4,7 & 9,6 & 2.29 \\
\hline Hymenaea courbaril L. & 4 & 3 & 0.36 & 1.30 & 0.53 & 5,9 & 13,7 & 2.19 \\
\hline
\end{tabular}

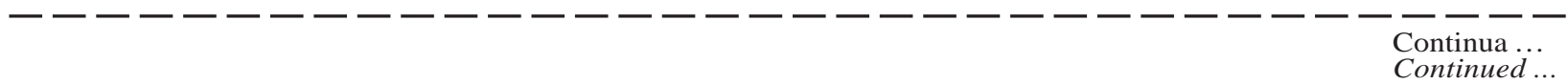

Revista Árvore, Viçosa-MG, v.37, n.4, p.577-585, 2013 
Tabela 1 - Cont.

Table 1 - Cont

\begin{tabular}{|c|c|c|c|c|c|c|c|c|}
\hline Espécies & $\mathrm{Ni}$ & $\mathrm{Na}$ & DR & DoR & FR & $\begin{array}{l}\text { Altura } \\
\text { média }\end{array}$ & $\begin{array}{l}\text { Diâmetro } \\
\text { médio }\end{array}$ & VI \\
\hline Eugenia aurata O.Berg. (252) & 9 & 5 & 0.81 & 0.49 & 0.88 & 3,4 & 6,4 & 2.18 \\
\hline Cordia cf naidophila I. M. Johnst. & 7 & 6 & 0.63 & 0.34 & 1.05 & 3,4 & 6,2 & 2.02 \\
\hline Guapira noxia (Netto) Lundell & 6 & 5 & 0.54 & 0.44 & 0.88 & 3,8 & 7,1 & 1.86 \\
\hline Vatairea cf macrocarpa (Benth.) Ducke & 6 & 5 & 0.54 & 0.40 & 0.88 & 4,2 & 6,7 & 1.82 \\
\hline Gomidesia cf affinis (Cambess.) D. Legrand & 7 & 5 & 0.63 & 0.27 & 0.88 & 3,1 & 5,3 & 1.77 \\
\hline Bowdichia virgilioides Kunth. (215) & 6 & 3 & 0.54 & 0.67 & 0.53 & 6,1 & 9,7 & 1.74 \\
\hline $\begin{array}{l}\text { Astronium fraxinifolium Schott ex Spreng. } \\
\text { Terminalia brasiliensis (Cambess. ex A. St.- Hil.) }\end{array}$ & 5 & 5 & 0.45 & 0.31 & 0.88 & 3,6 & 6,8 & 1.64 \\
\hline Eichler (331) & 6 & 4 & 0.54 & 0.38 & 0.70 & 3,8 & 6,4 & 1.63 \\
\hline Byrsonima verbascifolia (L.) DC. (258) & 5 & 4 & 0.45 & 0.41 & 0.70 & 4,3 & 7,0 & 1.56 \\
\hline Anadenanthera falcata (Benth.) Speg. (344) & 4 & 3 & 0.36 & 0.55 & 0.53 & 5,1 & 9,5 & 1.43 \\
\hline Platypodium elegans Vogel (226) & 4 & 4 & 0.36 & 0.32 & 0.70 & 4,5 & 7,2 & 1.38 \\
\hline Samanea tubulosa (Benth.) Barneby \& J.W. Grimes & 4 & 2 & 0.36 & 0.55 & 0.35 & 4,8 & 9,5 & 1.26 \\
\hline Terminalia argentea Mart.(398) & 4 & 4 & 0.36 & 0.16 & 0.70 & 4,0 & 5,4 & 1.22 \\
\hline Simarouba versicolor A. St.-Hil. & 4 & 3 & 0.36 & 0.32 & 0.53 & 4,6 & 7,7 & 1.21 \\
\hline $\begin{array}{l}\text { Schinus terenbithifolia Raddi } \\
\text { Tocoyena formosa (Cham. \& Schltdl.) }\end{array}$ & 4 & 3 & 0.36 & 0.31 & 0.53 & 4,1 & 7,7 & 1.20 \\
\hline K. Schum. (379) & 4 & 2 & 0.36 & 0.46 & 0.35 & 4,1 & 9,2 & 1.17 \\
\hline Myracrodruon urundeuva Allemão & 4 & 4 & 0.36 & 0.10 & 0.70 & 3,4 & 4,5 & 1.17 \\
\hline Eugenia bimarginata DC. (366) & 4 & 4 & 0.36 & 0.10 & 0.70 & 2,6 & 4,5 & 1.16 \\
\hline Terminalia fagifolia Mart. (271) & 3 & 1 & 0.27 & 0.67 & 0.18 & 5,7 & 13,5 & 1.11 \\
\hline Banisteriopsis laevifolia (A. Juss) B. Gates (247) & 4 & 3 & 0.36 & 0.14 & 0.53 & 3,0 & 4,9 & 1.03 \\
\hline Aspidosperma australe Müll. Arg. & 3 & 3 & 0.27 & 0.22 & 0.53 & 3,7 & 7,3 & 1.02 \\
\hline Miconia albicans (Sw) Steud. (339) & 3 & 3 & 0.27 & 0.16 & 0.53 & 3,2 & 6,4 & 0.96 \\
\hline Sloanea guianensis (Aubl.) Benth. (243) & 1 & 1 & 0.09 & 0.60 & 0.18 & 6,0 & 22,6 & 0.87 \\
\hline Copaifera langsdorffii Desf. (245) & 3 & 3 & 0.27 & 0.04 & 0.53 & 2,2 & 3,3 & 0.84 \\
\hline Huntheria $s p$ & 2 & 2 & 0.18 & 0.25 & 0.35 & 5,0 & 9,6 & 0.78 \\
\hline Austroplenckia populnea (Reissek) Lundell & 2 & 2 & 0.18 & 0.24 & 0.35 & 4,3 & 9,2 & 0.77 \\
\hline Ixora sp (287) & 2 & 2 & 0.18 & 0.23 & 0.35 & 2,8 & 8,4 & 0.76 \\
\hline Jacaranda mutabilis Hassl. (224) & 2 & 2 & 0.18 & 0.11 & 0.35 & 4,0 & 6,4 & 0.64 \\
\hline Machaerium acutifolium Vogel (317) & 1 & 1 & 0.09 & 0.26 & 0.18 & 7,0 & 15,0 & 0.53 \\
\hline Nectandra membranacea (Sw.) Griseb. & 2 & 1 & 0.18 & 0.14 & 0.18 & 2,3 & 7,0 & 0.50 \\
\hline Campomanesia sp (386) & 2 & 1 & 0.18 & 0.14 & 0.18 & 3,5 & 7,6 & 0.50 \\
\hline Miconia prasina (Sw.) DC. & 2 & 1 & 0.18 & 0.08 & 0.18 & 3,3 & 5,6 & 0.43 \\
\hline Mouriri guianensis Aubl. (327) & 2 & 1 & 0.18 & 0.07 & 0.18 & 3,0 & 5,1 & 0.42 \\
\hline Psidium guianense Pers.(427) & 1 & 1 & 0.09 & 0.14 & 0.18 & 4,0 & 10,8 & 0.40 \\
\hline Dilodendron bipinnatum Radlk. & 2 & 1 & 0.18 & 0.04 & 0.18 & 3,5 & 4,1 & 0.40 \\
\hline Casearia gossypiosperma Briq. (286) & 2 & 1 & 0.18 & 0.03 & 0.18 & 4,0 & 3,3 & 0.38 \\
\hline Rudgea sp (378) & 1 & 1 & 0.09 & 0.12 & 0.18 & 4,0 & 9,9 & 0.38 \\
\hline Connarus suberosus Planch. & 1 & 1 & 0.09 & 0.07 & 0.18 & 5,0 & 7,6 & 0.33 \\
\hline Matayba guianensis Aubl. (426) & 1 & 1 & 0.09 & 0.05 & 0.18 & 5,0 & 6,4 & 0.31 \\
\hline Tapirira guianensis Aubl. (432) & 1 & 1 & 0.09 & 0.05 & 0.18 & 5,0 & 6,4 & 0.31 \\
\hline Byrsonima basiloba A. Juss. & 1 & 1 & 0.09 & 0.04 & 0.18 & 2,0 & 6,1 & 0.31 \\
\hline Protium heptaphyllum (Aubl.) Marchand (402) & 1 & 1 & 0.09 & 0.03 & 0.18 & 3,0 & 5,1 & 0.30 \\
\hline Bauhinia longifolia (Bong.) Steud. & 1 & 1 & 0.09 & 0.03 & 0.18 & 3,5 & 4,7 & 0,29 \\
\hline Vernonia ferruginea Less. (330) & 1 & 1 & 0.09 & 0.02 & 0.18 & 2,0 & 4,5 & 0.29 \\
\hline Bauhinia rufa Graham & 1 & 1 & 0.09 & 0.02 & 0.18 & 4,0 & 4,1 & 0.29 \\
\hline Licania kunthiana Hook. f. & 1 & 1 & 0.09 & 0.02 & 0.18 & 1,7 & 4,1 & 0.29 \\
\hline
\end{tabular}

$\mathrm{Ni}$ (número de indivíduos), $\mathrm{Na}$ (número de ocorrência nas parcelas), DR (densidade relativa), DoR (dominância relativa), FR (freqüência relativa), Altura média, diâmetro médio, Vi (valor de importância. 
Tabela 2 - Comparação do índice de diversidade $\left(\mathrm{H}^{`}\right)$, equabilidade $\left(\mathrm{J}^{\prime}\right)$, número de espécies (N sp), número de famílias ( $\mathrm{N}$ fam), valor de importância (VI) para as 10 primeiras espécies e densidade total (DT) entre a área de estudo e outros trabalhos realizados em áreas de cerrado stricto senso.

Table 2 - Comparative of diversity index $\left(H^{\prime}\right)$, equability $\left(J^{\prime}\right)$, number of species $(N$ sp), number of families ( $N$ fam), importance value (VI) for the first 10 species and total density (DT) in the studied area and of other studies carried out in Cerrado senso stricto.

\begin{tabular}{|c|c|c|c|c|c|c|c|}
\hline Áreas de estudo & Critério & $\mathrm{H}^{\prime}$ & $\mathrm{J}$ & $\mathrm{N}$ sp & $\mathrm{N}$ fam & $\% \mathrm{VI}$ & DT \\
\hline Presente estudo - MS & $10 \mathrm{CAP}$ & 3,86 & 0,86 & 88 & 33 & 39,4 & 3690 \\
\hline Poconé - MT (SILVEIRA et al., 2000) & $10 \mathrm{CAP}$ & 1,31 & 0,86 & 47 & 25 & - & - \\
\hline Água Boa -MT (FELFINI et al., 2002) & $\mathrm{DAS} \geq 5 \mathrm{~cm}$ & 3,69 & 0,84 & 80 & 34 & 44,4 & 990 \\
\hline \multicolumn{8}{|l|}{ Nova Xavantina - MT (MARIMON JUNIOR.; } \\
\hline HARIDASAN, 2005) & DA30 $\geq 5 \mathrm{~cm}$ & 3,78 & 0,87 & 77 & 38 & 53,56 & 1890 \\
\hline \multicolumn{8}{|l|}{ Santo Antônio do Leverger - MT } \\
\hline (BORGES E SHEPHERD, 2005) & DAS $\geq 8 \mathrm{~cm}$ & 3,75 & - & 90 & 45 & 49,4 & 1653 \\
\hline Luziânia - GO (MEDEIROS et al., 2005) & DA30 $\geq 5$ & 3,60 & 0,87 & 61 & 35 & 44,1 & 573 \\
\hline Caldas Novas - GO (SILVA et al., 2002) & $\mathrm{DAS} \geq 13 \mathrm{~cm}$ & - & - & 67 & 29 & 56,7 & 1907 \\
\hline Chapada da Pratinha -DF (FELFINI et al., 1993) & DAS $\geq 5 \mathrm{~cm}$ & 3,62 & - & 80 & 30 & - & 1394 \\
\hline Paraopeba - MG (BALDUÍNO et al., 2005) & DA30 $\geq 5 \mathrm{~cm}$ & 3,57 & 0,83 & 73 & 38 & 127,0 & 1990 \\
\hline Senador Modestino - G (NERI et al., 2007) & $\mathrm{DAS} \geq 10 \mathrm{~cm}$ & 3,61 & & 91 & 38 & 49,32 & 6476 \\
\hline
\end{tabular}

As famílias com maiores riquezas foram Fabaceae (12), Myrtaceae e Caesalpinaceae (sete espécies cada), seguidas por Combretaceae e Rubiaceae (cinco espécies cada), constituindo juntas 40,4\% do total das espécies amostradas (Figura 2). Entre as famílias monotípicas (15 famílias), destacaram-se Lythraceae, Loganiaceae, Opiliaceae e Clusiaceae com, respectivamente, 69, 18, 16 e 16 indivíduos cada.

As famílias mais abundantes foram Vochysiaceae, Fabaceae, Myrtaceae e Erythroxylaceae com, respectivamente, 164, 138, 108 e 98 indivíduos (Figura 2). De forma geral, essas quatro famílias são bem representadas em áreas de Cerrado no Brasil Central (GOODLAND, 1970; RATTER et al., 1997; FELFILI et al., 2002).

As 10 espécies mais importantes foram Qualea grandiflora, Lafoensia pacari, Magonia pubescens, Qualea parviflora, Erythroxylum deciduum, Callisthene fasciculata, Myrcia fallax, Erythroxylum suberosum, Callisthene minor e Acosmium subelegans que, juntas, somaram 558 indivíduos, ou seja, 50,4\% do total amostrado e 44\% da área basal total (Tabela 1).

Entre as espécies com ampla ocorrência na área amostrada, destacam-se Qualea grandiflora (maior frequência relativa), Erythroxylum suberosum, Lafoensia pacari, Qualea parviflora e Myrcia fallax, entre outras. Das que apresentaram maior densidade, destacam-se Magonia pubescens, Lafoensia pacari e Erythroxylum deciduum, e as de maior dominância foram novamente Qualea grandiflora, Lafoensia pacari e Magonia pubescens.

Considerando as 88 espécies amostradas, 20 delas ocorreram em apenas uma parcela, 13 apresentaram um único indivíduo e 27 exibiram entre dois e cinco indivíduos (Tabela 1). Com esses dados, pode-se afirmar que cerca de $50 \%$ das espécies amostradas são raras numericamente na área.

Entre as espécies que apresentaram poucos indivíduos, ressalta-se Curatella americana (6 indivíduos), $H$. courbaril (4), Copaifera langsdorffii (3), geralmente bem representadas em áreas de Cerrado, sendo que Curatella americana apresentou lugar de destaque em VI na maioria dos trabalhos analisados (FELFILI et al., 2002; SILVA et al., 2002; BALDUÍNO et al., 2005). Esse fato pode estar associado às perturbações antrópicas passadas, visto que estas são espécies com grande potencial de exploração, seja para fins madeireiros, alimentícios ou medicinais.

As alturas e diâmetros médios das populações foram, respectivamente, $4,01 \mathrm{~m} \mathrm{e} \mathrm{7,26} \mathrm{cm}$. A análise da distribuição das classes de altura na comunidade evidencia que quase a metade dos indivíduos (546) compõe o estrato entre 2,0 e 4,0 m e está representado por várias espécies, das quais se destacam Davilla elliptica, Casearia sylvestris, Erythroxylum deciduum, Erythroxylum suberosum, Annona crassifólia e Genipa americana, entre outras. 
Das espécies com altura superior a $8 \mathrm{~m}$, destacaram-se Qualea grandiflora, Magonia pubescens, Callisthene fasciculata e Hymenaea stigonocarpa.

Através da análise da distribuição dos indivíduos em classes de diâmetro (Figura 3), pode-se verificar que a área de Cerrado stricto senso apresenta mais de 35\% dos seus indivíduos (407) com diâmetro abaixo de 5,1 cm e mais de 70\% dos indivíduos com diâmetro abaixo de 9,1 cm. Assim, pode-se afirmar que essa área se encontra em regeneração, uma vez que possui número alto de indivíduos por parcela, com diâmetros bastante reduzidos. Entretanto, a maior presença de indivíduos nas primeiras classes de diâmetro é um padrão frequente nas áreas de Cerrado stricto senso.

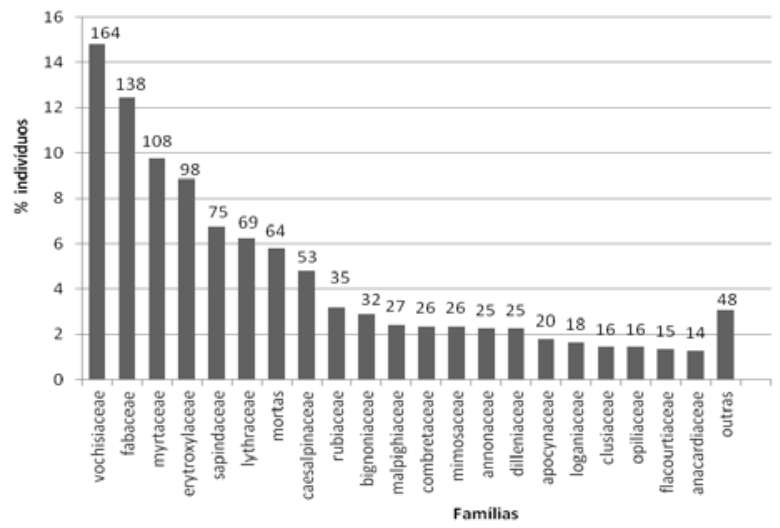

Figura 2 - Porcentagem e número de indivíduos para as principais famílias da área de cerrado stricto senso, município de Aquidauana.

Figure 2 - Percentage and number of individuals of the main families of Cerrado senso stricto in AquidauanaMS.

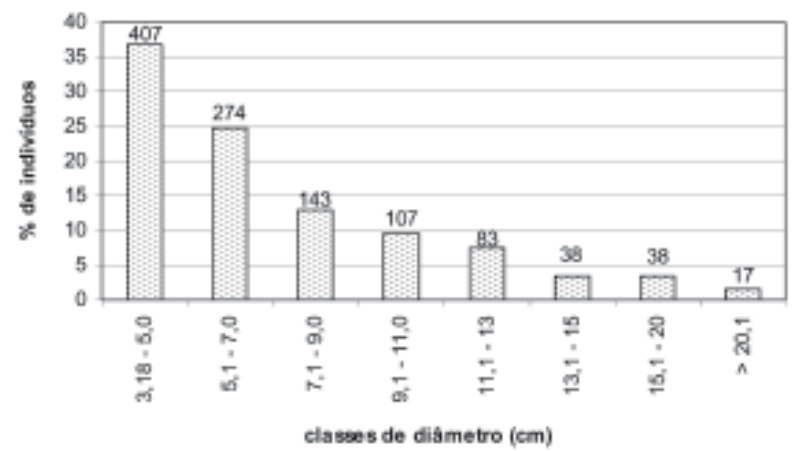

Figura 3 - Porcentagem de indivíduos em classes de diâmetro (m) numa área de cerrado stricto senso de AquidauanaMS.

Figure 3 - Percentual of individuals in diameter class ( $m$ ), area of Cerrado senso stricto in Aquidauana-MS.
Comparando a estrutura da área estudada com outros trabalhos realizados em Cerrado stricto senso (FELFILI et al., 1993; SILVEIRA et al., 2000; FELFILI et al., 2001; SILVA et al., 2002; BALDUÍNO et al., 2005; BORGES; SHEPHERD, 2005; MARIMON JR.; HARIDASAN, 2005; MEDEIROS et al., 2005; NERI et al., 2007), tem-se que essa estrutura apresentou a maior diversidade $\left(\mathrm{H}^{`}\right)$, a segunda maior densidade (DT) e a terceira maior riqueza ( $\mathrm{N}^{\circ} \mathrm{sp}$.) quando comparada com outros estudos (Tabela 2). Esse fato, aliado à menor porcentagem de VI nas espécies, confirma a alta heterogeneidade da vegetação de Cerrado local, alta diversidade e evidencia uma distribuição não restrita a poucas espécies, como ocorre em várias áreas de Cerrado, onde pequeno grupo de espécies compõe mais de 50\% do valor de importância da área (FELFILI et al., 1993).

A conservação das áreas naturais depende de inúmeros fatores, como tamanho do fragmento, riqueza e distribuição das espécies, grau de perturbação e pressão antrópica, entre outros.

A área analisada mostrou-se capaz de se recuperar naturalmente através da retirada dos principais fatores de pressão: acesso do gado ao local (isolamento da área com cercas), proibição da retirada de madeira nativa e não ocorrência de fogo nos últimos cinco anos. Alia-se a esses fatos a presença de propágulos na área. As espécies amostradas apresentam, em sua maioria, ampla distribuição em áreas de Cerrado stricto senso e não foram observadas espécies endêmicas. Entretanto, a escassez numérica (menos de cinco indivíduos) de aproximadamente 50\% das espécies amostradas requer atenção com relação ao tamanho do fragmento.

Ratter et al. (1997) enfatizaram que a expressiva heterogeneidade florística do Cerrado deve ser considerada em programas de conservação, sendo necessário proteger muitas áreas para conseguir preservar toda a sua biodiversidade.

Assim, com os dados obtidos neste estudo, pode-se ressaltar a importância do estabelecimento de uma unidade de conservação no local, visto que este pode constituir importante corredor entre a planície e o planalto pantaneiro, dada a sua localização, além de apresentar alta riqueza e diversidade, em comparação com outras áreas da Região Centro-Oeste.

Revista Árvore, Viçosa-MG, v.37, n.4, p.577-585, 2013 


\section{REFERÊNCIAS}

AGUIAR, L. M. S.; MACHADO, R. B.; MARINHO-FILHO, J. A diversidade biológica do cerrado. In: AGUIAR, L. M. S.; CAMARGO, A. J. A. (Ed.). Cerrado: ecologia e caracterização. Planaltina: Embrapa Cerrados, 2004. p.17-40.

BALDUÍNO, A. P. C. et al. Fitossociologia e análise comparativa da composição florística do cerrado da flora de Paraopeba-MG. Revista Árvore, v.29, n.1, p.25-34, 2005.

BORGES, H. B. N.; SHEPHERD, G. J. Flora e estrutura do estrato lenhoso numa comunidade de cerrado em Santo Antonio do Leverger, MT, Brasil. Revista Brasileira de Botânica, v.28, n.1, p.61-74, 2005.

BRASIL. Ministério do Meio Ambiente, dos Recursos Hídricos e da Amazônia Legal/ Plano de Conservação da Bacia do Alto Paraguai - PCBAP/Projeto Pantanal. Diagnóstico dos meios físicos e bióticos. Brasília: PNMA, 1997. v.2. Tomo II. 394p.

BRASIL. Ministério das Minas e Energia, Secretaria Geral. Projeto RADAMBRASIL: Levantamento de recursos naturais. Rio de Janeiro: 1990. v. 26.

EMPRESA BRASILEIRA DE PESQUISA AGROPECUÁRIA - EMBRAPA. Embrapa cerrados e a região dos cerrados: informações básicas e dados estatísticos. Planaltina: 1998. 24p.

FELFILI, J. M. et al. Composição florística e fitossociologia do cerrado sentido restrito no município de Água Boa-MT. Acta Botanica Brasilica, v.16, n.1, p.103-112, 2002.

FELFILI, J. M. et al. Fitossocilogia da vegetação arbórea. In: FELFILI, J. M.; SILVA JÚNIOR, M. C. Biogeografia do bioma cerrado: estudo fitofisionômico na Chapada do Espigão Mestre do São Francisco. Brasília: Universidade de Brasília, 2001. 152p.

FELFILI, J. M. et al. Análise comparativa da florística e fitossociologia da vegetação arbórea do cerrado sensu stricto na Chapada Pratinha, Brasil.

Acta Botanica Brasilica, v.6. n.2, p.27-46, 1993.
GOODLAND, R. J. A. Plants of the cerrado vegetation of Brasil. Phytologia, v.20, n.1, p.57-78, 1970.

INSTITUTO BRASILEIRO DO MEIO AMBIENTE E DOS RECURSOS RENOVÁVEIS - IBAMA. Ecossistema - Cerrado. Disponível: (www.ibama.gov.br), acesso em: nov. de 2002.

MACHADO, R. B. et al. Estimativas de perda da área do Cerrado brasileiro. Brasília: Conservation International, 2004.

MARIMON JUNIOR, B. H.; HARIDASAN, M. Comparação da vegetação arbórea e características edáficas de um cerradão e um cerrado sensu stricto em áreas adjacentes sobre solo distrófico no Leste de Mato Grosso, Brasil. Acta Botânica Brasílica, v.19, n.4, p.913926. 2005.

MEDEIROS, M. B.; GUARINO, E. S. G.; SILVA, G. P. S. Fitossociologia de um trecho de cerrado sensu stricto na bacia do Rio Corumbá - área de influência direta do aproveitamento hidroelétrico Corumbá IV (GO). Brasília: Embrapa Recursos Genéticos e Biotecnologia, 2005.

MÜLLER-DOMBOIS, D. \& ELLENBERG, H. Aims and methods in vegetation ecology. New York: John Wiley \& Sons, 1974.

NERI, A. V. et al. Análise da estrutura de uma comunidade lenhosa em área de cerrado sensu stricto no município de Senador Modestino Gonçalves, Norte de Minas Gerais, Brasil.

Revista Árvore, v.31, n.1, p.123-134, 2007.

RATTER, J. A.; BRIDGEWATER, S. \& RIBEIRO, J. F. Analysis of the floristic composition of the Brazilian cerrado vegetation III: comparison of the woody vegetation of 376 areas.

Edinburgh Journal of Botany, v.60, n.1, p.57-109, 2003.

RATTER, J. A.; RIBEIRO, J. F. \& BRIDGEWATER, S. The brazilian cerrado vegetation and threats to its biodiversity. Annals of Botany, v.80, p.223-230, 1997. 
SILVA, L. O. et al. Levantamento florístico e fitossociológico em duas áreas de cerrado sensu stricto no Parque Estadual da Serra de Caldas Novas, Goiás. Acta Botanica Brasilica, v.16, n.1, p.43-53, 2002.

SILVA, M. P. et al. Distribuição e quantificação de classes de vegetação do Pantanal através de levantamento aéreo. Revista Brasileira de Botânica, v.23, n.2, p.143-152, 2000.
SILVEIRA, E. A.; BARROS, L. T. L. P.; ALMEIDA, N. N. Mapa de vegetação e uso do solo na região de Poconé - MT: caracterização florísitica e estrutural. In: SIMPÓSIO SOBRE RECURSOS NATURAIS E SÓCIO-ECONÔMICOS DO PANTANAL - os desafios do novo milênio, 3., 2000, Corumbá. Anais... Corumbá: 2000. p.1-28.

SHEPHERD, J. Fitopac1: manual do usuário. Campinas: UNICAMP, 1994. 88p. 
